- Early consideration of the likely final restoration and the maintenance of appearance and function are areas of primary concern in the management of hypodontia.

- Necessary restorative treatment will reflect the decision to accept, close or redistribute spacing resulting from the absence of teeth.

- Resin-bonded bridges and implant supported prostheses are the preferred definitive restorative option for the majority of hypodontia patients.

- Removable partial dentures can usefully restore appearance and function particularly as an interim measure for patients with severe hypodontia

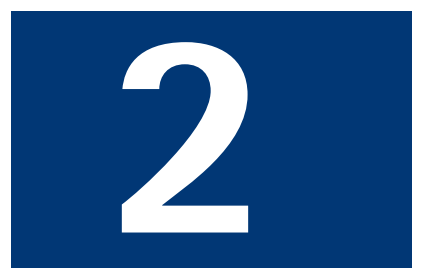

\title{
The interdisciplinary management of hypodontia: restorative dentistry
}

\author{
N. J. Jepson, ${ }^{1}$ F. S. Nohl, ${ }^{2}$ N. E. Carter, ${ }^{3}$ T. J. Gillgrass, ${ }_{1}^{4}$ J. G. Meechan, ${ }^{5}$ R. S. Hobson, ${ }^{6}$ and J. H. Nunn, ${ }^{7}$
}

This paper considers the role of restorative dentistry in the management of hypodontia. The paper describes the general restorative considerations common to patients with hypodontia and illustrates the variety of restorative techniques available in the restorative management of hypodontia and oligodontia.

THE INTERDISCIPLINARY

MANAGEMENT OF HYPODONTIA:

1. Paediatric dentistry

2. Restorative dentistry

3. Orthodontics

4. Oral surgery

5. The relationship between an interdisciplinary team and the general dental practitioner

\footnotetext{
Senior Lecturer/Honorary Consultant in Restorative Dentistry, ${ }^{3}$ Consultant in Orthodontics, ${ }^{5}$ Senior Lecturer/Honorary Consultant in Oral Surgery, ${ }^{6}$ Senior Lecturer/Honorary Consultant in Orthodontics, School of Dental Sciences, Framlington Place, Newcastle upon Tyne NE2 4BW: ${ }^{2}$ Consultant in Restorative Dentistry, Newcastle Dental Hospital, Richardson Road, Newcastle upon Tyne NE2 4AZ; ${ }^{4}$ Consultant in Orthodontics, Edinburgh Postgraduate Institute, Lauriston Building, Lauriston Place, Edinburgh, EH3 9YW, Scotland; ${ }^{7}$ Professor and Head of Department, Department of Public and Child Dental Health, Dental

School and Hospital, Lincoln Place, Dublin 2, Ireland

Correspondence to: Dr Nick Jepson,

Department of Restorative Dentistry, The Dental School, Framlington Place,

Newcastle upon Tyne NE2 4BW

E-mail:n.j.a.jepson@ncl.ac.uk
}

\section{Refereed Paper}

๑ British Dental Journal 2003; 194: 299-304
The principal aims in the restorative management of patients with hypodontia, the restoration and maintenance of function and appearance, differ little from that of any other patient. So too does the variety of treatment modalities used to restore the dentition of these patients. The difference and the challenge lie in the application of these principles in the long-term management of patients with congenitally missing teeth. These are reflected in general management issues such as age, timing of intervention and the severity of the hypodontia but, above all, the need to co-ordinate restorative treatment within a treatment plan that frequently involves a number of specialities.

General aspects of the interdisciplinary management of hypodontia have been described in Part 1 of this series. This article will describe the general considerations common to the restorative management of patients with congenitally missing teeth and illustrate the variety of restorative techniques available in the restorative management of hypodontia and oligodontia.

\section{GENERAL RESTORATIVE CONSIDERATIONS}

The treatment options available for the management of young hypodontia patients are essentially those available for the replacement of missing teeth in adults and will include removable partial dentures, conventional and adhesive bridges and implant supported prostheses. Clearly both the timing and manner of their application must reflect the needs and limitations imposed by a young, growing individual:
- The need to await eruption and root formation of permanent teeth frequently delays restorative intervention.

- Adhesive rather than conventional bridgework is generally preferred as this avoids preparation of unrestored teeth, with the large pulp chambers commonly found in young individuals.

- Short clinical crowns of recently erupted permanent teeth and, in particular, retained primary teeth, can compromise the retention of both adhesive and conventional bridgework.

- Placement of implant-supported prostheses is unlikely to be considered until clinical signs of growth cessation are present.

- Other general factors include the ability of the patient to accept treatment; examination, work and social commitments and peer pressure.

Within the interdisciplinary management of hypodontia, the areas of primary concern to the restorative dentist include:

- Early consideration of the likely final restoration

- Maintenance of appearance and function.

\section{Early consideration of the likely final} restoration

Early visualisation of the likely definitive longterm restoration of missing teeth is essential to the satisfactory management of patients with hypodontia. An understanding of the final aim of treatment is important to the development of confidence and co-operation of both child and 

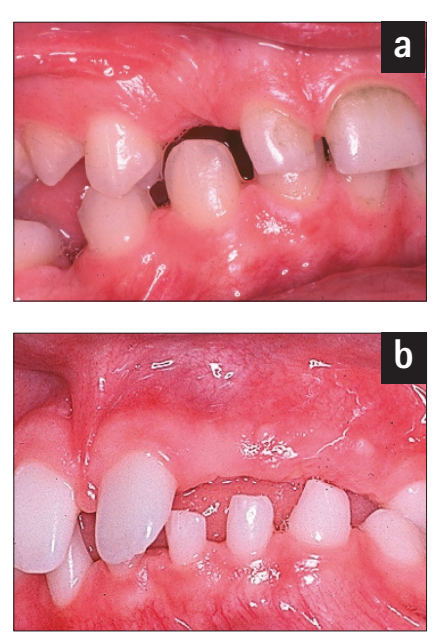

Fig. 1 (a) Over eruption of the unopposed LR3 and (b) LLC. There is a reduction in the available vertical space that in (a) will also cause problems in lateral excursive movements. Both cases demonstrate increased overbite - a common feature in hypodontia patients
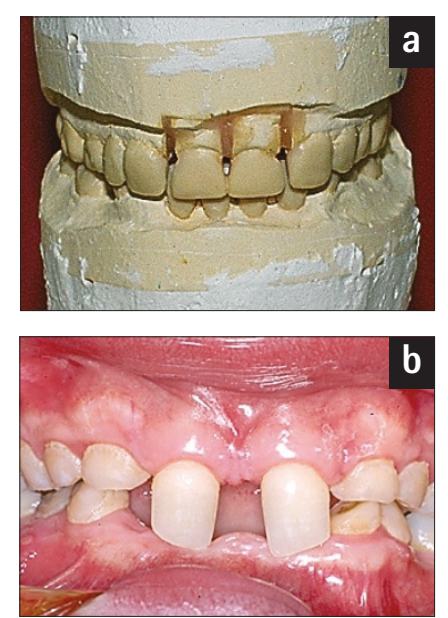

Fig. 2 (a) Diagnostic wax-up of the intended treatment outcome for the patient illustrated in (b). This will allow a good assessment of the aesthetic possibilities and will give a clear indication to the orthodontist of the necessary tooth movements. They will very usefully serve to inform and involve the patient over a likely prolonged course of treatment parents and goes some way towards anticipating requests for early restorative intervention when this is not appropriate. Clinically, it allows for the development of an overall treatment strategy and, in particular, a consideration of the space required to optimise both the appearance and occlusion of the replacement teeth. Unopposed permanent teeth, or those opposed by worn primary teeth, may overerupt and restrict the vertical space available for the planned restoration (Fig. 1). This will be apparent in the intercuspal position but can also cause problems during excursive movements, when over-erupted teeth are found to drive through an edentulous space. A bridge pontic or an implant supported crown as the sole excursive contact could be subject to significant non-axial loads and may be at risk of mechanical overload, leading to failure of prosthetic components or osseointegration. Careful planning by the interdisciplinary team to assess the aesthetic and occlusal possibilities of restorative treatment is therefore essential. This will frequently require diagnostic wax-ups and tooth set-ups using a semi-adjustable articulator where indicated (Fig. 2). This understanding will inform orthodontic treatment necessary to position the remaining, and in particular, abutment teeth in an optimal position. Such planning may indicate that a particular advantage could be gained by the transplantation of an ectopic tooth to act as an additional abutment. This can be especially useful when several anterior teeth are missing. Auto transplantation will be discussed further in Part 4 of this series.

In general terms, fixed restorations are preferable to removable ones as the definitive restoration. This reflects both the reduced willingness of younger patients to accept dentures and the strong evidence indicating the potential of removable prostheses to harm the remaining teeth over the long-term. Increasingly, implantsupported prostheses are considered the preferred definitive replacement for both single and many missing teeth. Irrespective of the option selected for the final restoration, the space requirements aesthetically and occlusally are similar. It is therefore advisable to create enough space mesio-distally and vertically for any of the treatment options under consideration. A failure to do so may restrict the choice for the final restoration.

\section{Maintenance of appearance and function}

As has been intimated above, the definitive replacement of missing teeth is frequently delayed until the eruption of permanent teeth and/or any necessary tooth movement has been completed. In the case of implant-supported prostheses, treatment will not usually commence until growth has ceased. In the interim, there may be aesthetic and functional demands by the patient that can be met by the provision of removable partial dentures. This is commonly the case for patients with severe hypodontia where the use of simple mucosal borne and overlay partial dentures to replace teeth and restore occlusal face height can effect a considerable improvement to appearance and function (Fig. 3). Such prostheses may also act as space maintainers following completion of active orthodontic treatment. Where further growth and/or tooth eruption is anticipated, the denture will normally be all acrylic to allow for easy adjustment and replacement, though denture failure and loss is a potential disadvantage. ${ }^{1}$ In the majority of cases, sufficient improvement can be made through the provision of an upper denture only. This facilitates the use of a design that will avoid unnecessary tissue damage and improves patient acceptance. Well-designed removable partial dentures with a cast cobaltchromium framework can serve well as a replacement for missing teeth in teenagers and young adults.

Provision of interim removable partial dentures should be avoided if at all possible and should only be considered in response to a specific complaint by the patient or when there is a clinical need; such as space maintenance and the prevention of over-eruption of teeth. Their use in response to the understandable concerns and demands of parents can often lead to failure through a lack of compliance by the child during treatment and in the subsequent non-use of the denture.

\section{MANAGEMENT OF HYPODONTIA}

The successful treatment of patients with the congenital absence of one or a few teeth strongly demonstrates the advantages of early diagnosis and interdisciplinary co-operation, typically between the orthodontist and restorative dentist. There have been a number of reports describing the specific and commonly occurring absence of missing maxillary lateral incisors. ${ }^{2,3}$ The principles of treatment outlined in these reports apply to all cases of hypodontia.

In broad terms, the necessary treatment reflects the pattern of tooth absence, the amount of residual spacing, existing malocclusions and patient attitudes. The following options are available:

\section{- Accept spacing}

- Space closure

- Redistribution of space

\section{Accept spacing}

Minimal spacing resulting from the absence of teeth may not be an aesthetic concern to patients or they may be unwilling to accept necessary corrective orthodontic and restorative procedures. This is particularly true where spacing is posterior to the canine or is not seen when smiling. Anteriorly, the appearance of a diastema and malformed teeth can be improved using bonded composite build-ups and veneers (Fig. 4). Diagnostic wax-ups of the likely outcome are of value in informing the patient and planning individual tooth restorations. 


\section{Space closure}

Space closure and modification of the canine to resemble a lateral incisor is a common treatment option where maxillary lateral incisors are missing. Space closure may be allowed to occur spontaneously or is controlled through active orthodontic treatment. The influence of any underlying malocclusion on orthodontic extraction choices and treatment will be described in Part 3 of this series. Treatment planning for these cases should include a trial diagnostic setup to confirm the possibilities of a functional and aesthetic result.

An important factor commonly influencing a decision to close space is the ability to modify the shape and colour of the canine to resemble a lateral incisor (Fig. 5). Proximal enamel reduction to reduce tooth width and produce more vertical proximal surfaces, particularly mesially, is normally required. The majority of this reduction should be made mesially and should be undertaken before final space closure. A flattened incisal edge is produced by reduction of the canine tip and the addition of composite to the mesial and distal corners. This is normally undertaken after completion of orthodontic tooth movement, as is the desirable reduction of the buccal contour. This reduction is however limited as over-reduction of enamel will darken the tooth as the dentine colour shows through. The canine already has a higher chroma than other anterior teeth but its shade can be improved subsequently with the use of vital tooth bleaching procedures. A further disadvantage of the use of the canine to mimic the lateral incisor is the more apical position of the gingival margin commonly associated with this tooth. Though acceptable with low smile lines, the discrepancy in the levels of the gingival margins between the canine and central incisors can be disagreeable when high smile lines reveal the gingival tissues. Canines in the positions of lateral incisors may also preclude the prosthodontic convenience of canine guidance in lateral excursions.

The major advantage of space closure is the avoidance of a permanent restoration to replace missing teeth. Apart from the minor restorative procedures described above, all treatment is completed at the end of orthodontic intervention and the need for maintenance is low. The permanence of the final result is debatable given the tendency to relapse following orthodontic space closure. However, such problems of tooth stability following orthodontic treatment apply also to techniques used to create and redistribute space. The implications of orthodontic retention on restorative treatment in the long term are considered further in a later section of this paper.

\section{Redistribution of space}

Where several teeth are congenitally absent the orthodontic redistribution of space to allow restoration with prostheses is frequently the treatment of choice. The aesthetic and functional possibilities of such an approach should be confirmed with a trial diagnostic set-up. This will, in particular, indicate the positions of potential abutments necessary to optimise the aesthetics and occlusion of the final restoration(s), information essential to effective orthodontic treatment. Other factors influencing the orthodontic management of space redistribution will be discussed in Part 3 of this series.

\section{RESTORATIVE OPTIONS}

Fixed restorations (resin-bonded and conventional bridges, implant supported prostheses) are the preferred treatment for such patients. Removable partial dentures have a place as temporary tooth replacements and space maintainers following completion of orthodontic treatment. However, their bulk and potential movement is functionally and socially unacceptable to many patients as a definitive restoration as is their potential to harm the remaining teeth.

\section{Resin-bonded bridges}

Improved techniques and materials have seen an increasing acceptance of resin-bonded bridges as a definitive restoration for one or two tooth spaces that are particularly suited to teenagers and young adults. Current research suggests an anticipated survival of over $80 \%$ for a period of 6 years or longer ${ }^{4-7}$ though this is subject to a number of variables. Past reports of a particularly poor survival in patients under 20 years of age $^{8}$ may be a result of the inadequacies of the then available techniques and materials but may also reflect the reduced bonding area available on 'immature' permanent teeth and, in particular, retained primary teeth. It may also be simply due to a more active lifestyle of such patients. ${ }^{9}$ In this regard, there is growing evidence of an improved survival when preparation of the tooth to improve resistance form and maximise the bonding area is undertaken. ${ }^{6,7,10}$ Such preparations include grooves, rest seats, guide planes and positive finishing lines. Though not to the extent associated with conventional bridgework, such preparation is irreversible and not of the minimally invasive nature originally associated with the technique.

Some of the design issues arising from the use of resin-bonded bridgework in hypodontia patients are illustrated in Figure 6. A cantilever design carrying a single pontic supported by a single abutment is increasingly preferred. Recent cohort studies indicate that the survival of cantilever resin-bonded bridges is at least as good as more conventional fixed-fixed designs. ${ }^{11}$ The use of a single abutment simplifies clinical procedures and, by allowing some flexibility in the choice of a path of insertion, can minimize the unsightly interproximal show of metal retaining wings. In the particular instance of the replacement of missing maxillary lateral incisors in young patients, use of the canine as the single abutment can avoid the greying effect caused by the show of metal retaining wings through the thin,
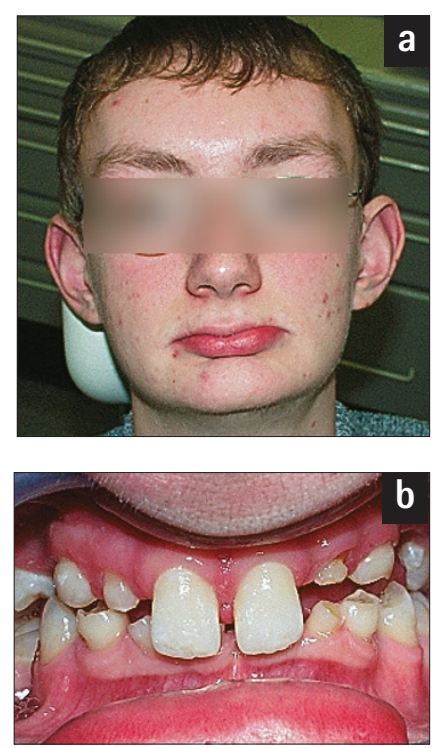

Fig. 3 A poor appearance caused by worn deciduous teeth, missing teeth and over closure was a concern to this 15-year-old boy (eyes obscured)
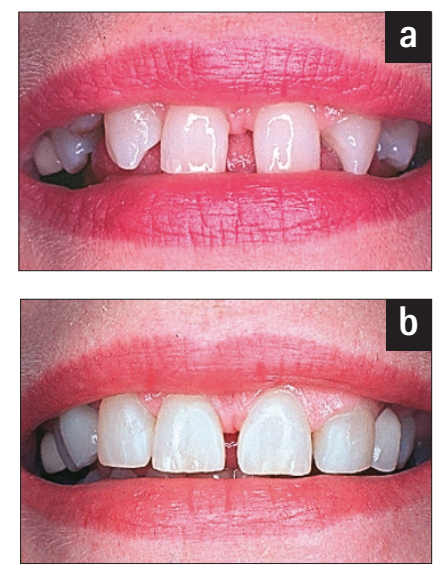

Fig. 4 Acceptance of spacing: the poor appearance resulting from spacing and the shape of UR3 and UL3 (a) has been corrected by a combination of tooth reshaping, adhesive composite additions and a minimal preparation dentine bonded crown on URC and a resin-bonded bridge at UL3(b) 

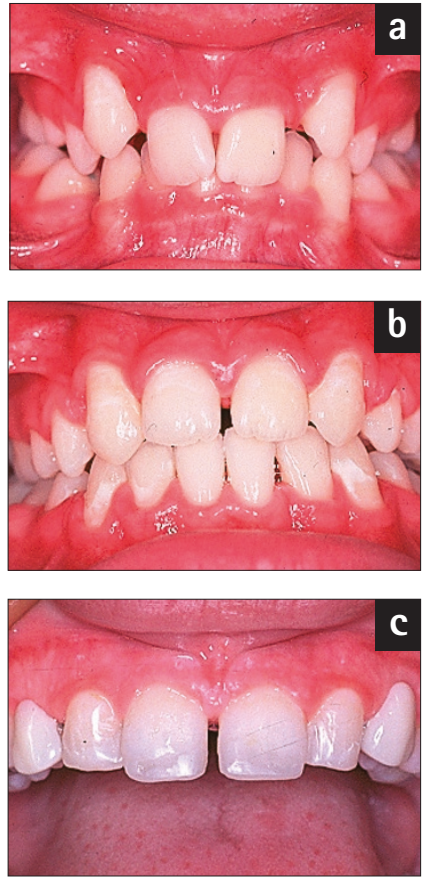

Fig. 5 Space closure: (a) and (b) orthodontic treatment to realign anterior segments and reduce overbite has resulted in an appearance that was acceptable to the patient. (c) Missing UR2 and UL2 - space closure and reshaping of anterior teeth through enamel reduction, composite additions and porcelain veneers translucent enamel of the central incisor. The refinement of all-porcelain adhesive bridges may overcome this difficulty. The use of double abutments for resin-bonded cantilever bridges is generally to be discouraged.

Tooth movements associated with the orthodontic redistribution of space are subject to relapse. Construction of resin-bonded bridges is not normally considered until completion of at least 6 months full time orthodontic retention with fixed or removable retainers. It is essential that effective retention is maintained during construction of the bridge and this may entail removal and replacement of retaining arch wires immediately before and after tooth preparation and impressions. The long-term stability of tooth positions in the absence of permanent retention is a matter of debate. Fixed-fixed designs may well provide the necessary retention but the same cannot be said of the preferred cantilever design where slippage of proximal contacts is possible. This may be overcome in part by broadening the contact. Additional retention through the use of a bonded flexible wire or the continued use of a night time removable retainer may be necessary. Figure 7 illustrates a typical case of space redistribution and restoration with resin-bonded bridges.

\section{Conventional bridges}

Use of conventional porcelain fused to metal bridgework is generally limited to the older patient and where resin-bonded bridgework is contra-indicated by the presence of large restorations. In the young patient, the necessary tooth reduction attracts the risk of pulp exposure and subsequent periapical pathology because of the large pulp. Although the reported survival of conventional bridgework is better than that of resin-bonded bridges, ${ }^{12}$ it is still short in relation to the expected life span of a young patient. Failure, commonly as a result of recurrent caries and apical involvement, can be catastrophic and may result in further tooth loss.
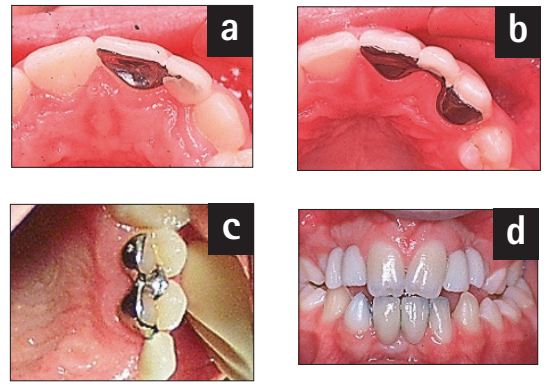

Fig. 6 The design of resin-bonded bridges in hypodontia patients. (a) A simple cantilever bridge is the preferred design but a fixed-fixed design (b) may be necessary if orthodontic tooth movements to create space for the prosthesis are not stable. Note the use of broad bonding areas for both designs. (c) Use of double abutments in an attempt to improve bridge retention is unnecessary and should be avoided. (d) 'Greying' of abutment teeth

\section{Implant supported prostheses}

General considerations

Several features of hypodontia make the provision of osseointegrated implants attractive but at the same time more challenging. Despite best efforts, compromises in final tooth and implant positions may be more likely to occur due to the limitations imposed by the dento-alveolar features of hypodontia discussed below. During the restorative phase of treatment, implant level impressions are particularly useful to facilitate the choice of abutment(s) and final prosthesis design, as well as enabling trial restorations to be constructed. Choosing and becoming familiar with an implant system which offers good surgical and restorative flexibility is an important consideration.

The ability to avoid using teeth as bridge abutments and the capacity to maintain appropriate interdental spaces are advantages of implant treatment common to any partially dentate case. Implants are particularly attractive in patients with a spaced, microdont dentition where minimal clinical crown height and small surface area make poor abutments for both conventional and adhesive bridgework.

Being 'ankylosed', osseointegrated implants have also been used as anchorage points for orthodontic tooth movements ${ }^{13}$ and are doubly advantageous if the implant can subsequently be used to support a restoration. In patients with anodontia or oligodontia, the use of strategically placed implants to retain and give stability to overdentures would appear to offer advantages over conventional prostheses. ${ }^{14,15}$

Patients with congenitally missing teeth usually present in childhood and the implications of placing implants in growing jaws will be discussed in Part 4 of this series. There are, however, other longitudinal considerations:

- Longer term crestal bone loss around the necks of implants range from $0.02 \mathrm{~mm}$ to $0.1 \mathrm{~mm}$ per year ${ }^{16}$ with a proposed cut-off for failure at 0.2 mm per year. ${ }^{17}$ The clinical implications of this rate of crestal bone loss may be insignificant in elderly patients. However, a rate of crestal bone loss of $0.1 \mathrm{~mm}$ per year from an early age could result in problems much later in life. Very long-term studies of implants placed in childhood are still awaited.

- Slow changes in the relative position of teeth and implants may also occur in adulthood. ${ }^{18}$

A lack of knowledge as to the certainty or relevance of these changes can lead to difficulties with informed consent. There is certainly a need for patients to understand the requirements for long-term maintenance and possible revisions of prosthesis design.

\section{Limitations imposed by the dento-alveolar features of hypodontia}

Narrow alveolar processes: Where no teeth have developed and at sites where small primary teeth (with no successors) have been retained, the 
alveolar ridge may be narrow (Fig. 8a). A discrepancy between the proposed tooth position, as judged by diagnostic wax-up or trial restoration, and an alveolus affected by horizontal tissue loss will be apparent. In such situations it might not be possible to install implants in a useful relationship to the proposed restoration, and within sufficient bone to provide the initial stability so critical for predictable osseointegration. ${ }^{19}$ Bone augmentation may be required as a preliminary procedure to circumvent the problem. Even when initial implant stability can be achieved at the time of placement, bone augmentation or ridge dilatation may still be required in order to avoid a significant dehiscence or fenestration at the implant surface.

The placement of narrow implants may seem attractive when faced with a narrow ridge. However, implant strength decreases significantly as diameter is reduced by only a small amount. ${ }^{20}$ Narrow implants used to replace wider teeth may also make adequate crown emergence profile difficult, especially if the implant head is shallow. As a general guide, implant diameter should be appropriate to the diameter of the tooth being replaced.

Where adequate alveolar bone dimensions exist at a more apical level, but where the ridge crest itself is narrow, it is sometimes possible to reduce the height of the ridge to a level where adequate bone width is present (Fig. 8b). However, this approach may result in an unfavourable crown: implant ratio as well as aesthetic and hygiene difficulties due to differing gingival levels between the implant bearing alveolus and neighbouring tissues.

Vertical alveolar defects: In the same way that loss of teeth leads to residual ridge resorption, failure of dental development may result in a significant deficit of alveolar bone height. This may leave inadequate bone to accommodate optimum implant length and increased risk of impinging on structures, such as the inferior alveolar neurovascular bundle and maxillary sinus, in an attempt to optimise length. In the absence of teeth, the posterior maxillary alveolus may also become 'pneumatised' with obvious implications for implant placement. An implant placed where there is a vertical alveolar deficit can necessitate a very long clinical crown with obvious aesthetic problems and unfavourable biomechanics caused by the high crown: implant ratio. The latter problems highlight the great benefit in retaining primary teeth in order to maintain alveolar bone for subsequent, carefully timed implant placement.

Mesio-distal tooth positions: Adequate mesio-distal space is needed not only for implant crowns, but also for the implant itself. Careful radiographic assessment of inter-root space at proposed implant sites is critical (Fig. $8 \mathrm{c})$. There is a need for the restorative dentist to work with the orthodontist to achieve optimum root angulation before implant therapy. Narrow implants and implants that taper like teeth may be helpful if space is limited, always taking into account the implications for implant strength. Figures 8d and 8e show how implant crowns have been used to replace missing upper lateral incisors.

\section{Post-orthodontic stability}

As previously discussed, the stability of tooth positions after orthodontic treatment may be problematic. In this regard, bridgework (by virtue of the retainers) offers the potential benefit of maintaining the position of abutment teeth. Stand-alone implant restorations do not offer the same advantage. As for cantilever bridgework, it may be possible to broaden interproximal contacts between implant crowns and adjacent teeth in an attempt to provide some resistance to the possibility of tooth rotation and slipped contacts. However, the latter option risks compromising the appearance of incisal and gingival embrasures. Other innovative designs linking teeth to implant superstructures may be possible. Further research and development in this area is required.

\section{OLIGODONTIA (SEVERE HYPODONTIA)}

The greater the number of missing teeth the greater is the likely need and demand for treatment. By the very nature of this condition, failure of eruption of several permanent and, at times, primary teeth should lead to early recognition by the primary care practitioner and the concerned parent. Indeed, early diagnosis is essential to the development of treatment strategies needed to manage the continuum of continued growth and development and varying aesthetic and functional needs of the patient until growth is complete and definitive restorations can be placed.

Restorative problems encountered in patients with congenital absence of many teeth reflect the quality of available alveolar bone and occlusal disturbance. The absence of teeth is often associated with a reduction in both the height and quality of the bone of the alveolar process. Preservation of alveolar bone by retain-
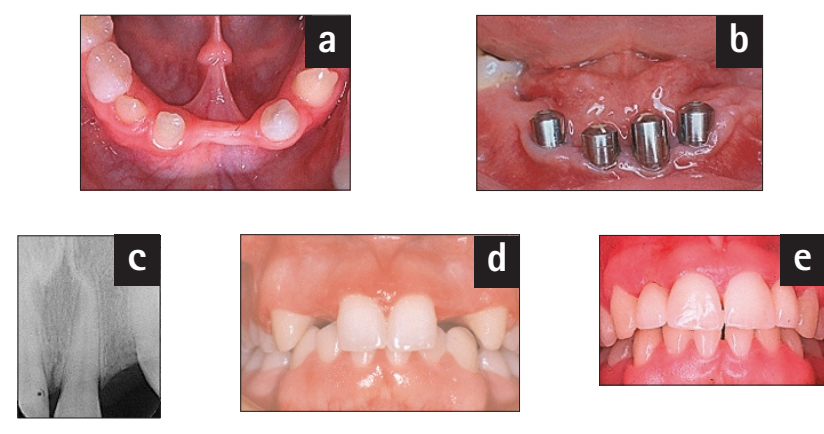

Fig. 8 Implant supported prostheses: (a) Narrow, underdeveloped alveolar ridge associated with LR1, LL1 and LL2 teeth; (b) a narrow ridge crest has been reduced in height to a level sufficiently wide to accommodate implants (healing abutments in situ); (c) the distal inclination of UL1 demonstrates the importance of careful radiographic assessment of mesial distal space at a proposed implant site; (d) and(e) this patient had orthodontic realignment to create space for implant crowns replacing the missing upper lateral incisors. The upper central incisors have also been widened using directly applied composite resin 
Hobkirk J A, Goodman J R, Reynolds I R. Component failure in removable partial dentures for patients with severe hypodontia. Int J Prosthodont 1989; 2: 327-330.

2. Millar B J, Taylor N G. Lateral thinking: The management of missing upper lateral incisors. Br Dent J 1995; 179: 99-106.

3. Sabri R. Management of missing maxillary lateral incisors.

JAm Dent Assoc 1999; 130: 80-84.

4. Creugars N H J, Kayser A F, van't Hof M A. A seven-and-a-half-year

survival study of resin-bonded bridges. J Dent Res 1992; 71: 1822 1825.

5. Rammelsberg P, Pospiech P, Gernet W. Clicial factors affecting adhesive fixed partial dentures: A 6 year study. J Prosthet Dent 1993; 70: 300-307.

6. Probster B, Henrich G M, Gutenberg J. 11-year follow up study of resin bonded fixed partial dentures. Int J Prosthodont 1997; 10: 259-268.

Behr M, Leibrock A, Stich W,

Rammelsberg P, Rosentritt M, Handel $\mathrm{G}$. Adhesive-fixed partial dentures in anterior and posterior areas. Clin Oral Investig 1998; 2: 31-35.

8. Dunne S M, Millar B J. A longitudinal study of the clinical performance of resin bonded bridges and splints. $\mathrm{Br}$ Dent J 1993; 174: 405-412.

9. Williams V D, Thayer K E, Denehy G E Boyer D B. Cast metal, resin-bonded prostheses: A 10 year retrospective study. J Prosthet Dent 1980; 61: 436-440.

10. De Kanter R J A M, Creugars N H J, Verzijden C W G J M, van't Hof M A. A five year multi practice clinical study on posterior resin bonded bridges. J Dent Res 1998; 77: 609-614.

11. Hussey D L, Linden G J. The clinical performance of cantilevered resinbonded bridgework. J Dent 1996; 24: 251-256.

12. Scurria M S, Bader J D, Shugars D A. Meta-analysis of fixed partial denture survival: Prostheses and abutments. J Prosthet Dent 1998; 79: 459-464.

13. Odman J, Lekholm U, Jemt T, Thilander B. Osseointegrated implants as orthodontic anchorage in the treatment of partially edentulous adult patients. Eur J Orthod 1994; 16: 187-201.

14. Kearns G, Sharma A, Perrott D, Schmidt B, Kaban L, Vargervik K. Placement of endosseous implants in children and adolescents with hereditary ectodermal dysplasia. Oral Surg Oral Med Oral Path 1999; 88: $5-10$. ing primary teeth is clearly advantageous but may be compromised by the rapid wear of opposed primary teeth and an unpredictable and, at times, rapid root resorption despite the absence of permanent successors. Attrition of retained lower primary incisors and the associated over-eruption of maxillary incisors is a common finding. Primary molars with no successors may become ankylosed with increasing infraocclusion and consequent tipping of adjacent teeth. Permanent teeth often erupt into poor positions and unopposed molar teeth tend to over-erupt. The resulting occlusion may be associated with a deep overbite, decreased interocclusal space and a reduced occlusal face height. This, in addition to large spaces where teeth are missing, may be a cause of complaint about appearance.

There is increasing evidence that implant supported prostheses are the preferred definitive restoration for patients with oligodontia. The treatment strategy is one of preparing the patient for the possibility of later placement of implant-supported prostheses and will commonly involve the following:

- Improvement and maintenance of appearance and function with simple removable partial dentures

- Orthodontic treatment to improve the position of erupted and guide erupting permanent teeth as well as reducing the overbite.

- Consideration of auto transplantation

- Preservation of residual alveolar bone.
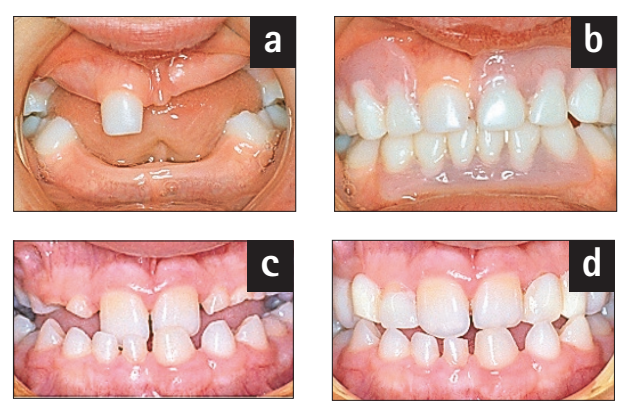

Fig. 9 Use of removable partial dentures in patients with oligodontia: (a) and (b) - Simple acrylic partial dentures to restore the appearance of a 3 year old patient following adverse peer comment when he began nursery school. Note the use of 'primary' denture teeth. (c) and (d) simple acrylic partial overlay denture to restore appearance and function. Primary teeth have been retained to maintain alveolar bone. This patient will eventually be considered for implant-supported prostheses
The early use of a simple acrylic partial denture or dentures to improve appearance in very young patients may be required in response to social and peer pressures associated in particular with the start of schooling (Fig. 9a and b). It should be emphasised that agreement to provide such treatment should be in response to the child's complaint and not in response to the natural concern of parents. Their construction will present some difficulties of patient management and will require imagination to tailor conventional denture techniques for use in the very young child. If possible, it is normally better to restrict treatment to one arch only - preferably the maxillary arch - but once the finished denture is fitted patient acceptance is often very good. An important factor in this acceptance is that the appearance of the denture should reflect the child's age; primary prosthetic teeth should be used for very young patients. Modification to existing dentures or their replacement will be required as the patient grows and permanent teeth erupt.

An increasing concern about appearance is common in teenage patients and simple partial dentures can effect a considerable improvement (Fig. 9c and 9d). Their provision should await the completion of any necessary orthodontic treatment for which they can act as an effective retainer. Where suitable teeth are available for support, for example retained primary teeth, overlay or overdenture designs should be used. These prostheses can provide important diagnostic information as to the effects on appearance of both the position of replacement of teeth and an increased occlusal face height.

\section{SUMMARY}

The restorative dentist has an important role to play in the overall management of the treatment of patients with hypodontia. Central to this role is an early consideration of the likely definitive replacement for missing teeth for which there is an increasing preference for the use of implantsupported prostheses. Partial dentures are commonly used as a temporary treatment to restore and maintain appearance and function. Resinbonded bridgework has an important role as both an interim and definitive replacement for single missing teeth. Close interdisciplinary working with, in particular, orthodontic colleagues is paramount.

The authors would like to thank MrD G Smith, Consultant in Restorative Dentistry, Newcastle Dental Hospital for his advice and help given in the preparation of this paper.
15. Durstberger G, Celar A, Watzek G. Implant-surgical and prosthetic rehabilitation of patients with multiple dental aplasia: A clinical report. Int J Oral Maxillofac Implants 1999; 14: 417 - 423

16. Bryant SR. The effects of age, jaw site, and bone condition on oral implant outcomes. Int J Prosthodont 1998; 11: 470-490.

17. Albrektsson T, Zarb G A, Worthington $P$, Eriksson A R. The long-term efficacy of curently used dental implants: A review and proposed criteria of success. Int J Oral Maxillofac Implants 1986; 1: 11-25.

18. Oesterle LJ, Cronin R J J. Adult growth, aging, and the singletooth implant. Int J Oral Maxillofac Implants 2000; 15: 252-260.

19. Meredith N. Assessment of implant stability as a prognostic determinant. Int J Prosthodont 1998; 11: 491-501.
20. Anon. Brånemarksystem ${ }^{\circledR}$ guidelines narrow platform. Nobel Biocare AB., 1996.

21. Bergendal $B$, Bergendal $T$, Hallonsten A L, Koch G, Kurol J, Kvint S. A multidisciplinary approach to oral rehabilitation with osseointegrated implants in children and adolescents with multiple aplasia. Eur J Orthod 1996; 18: 119-129. 